Proc. Indian Acad. Sci. (Chem. Sci.), Vol. 102, No. 5, October 1990, pp. 681-685.

(C) Printed in India

\title{
Laser Raman and infrared studies on hydrotropes and related materials
}

\author{
S B KARTHA ${ }^{1}$, V G JAIKAR ${ }^{2}$, M M SHARMA $^{2}$, and V B KARTHA ${ }^{1 *}$ \\ ${ }^{1}$ Spectroscopy Division, Bhabha Atomic Research Centre, Trombay, Bombay 400085, India \\ ${ }^{2}$ University Department of Chemical Technology, Matunga, Bombay 400019, India
}

\begin{abstract}
Hydrotropes, which are molecules used as solubilising agents in aqueous systems, are closely related to surfactants and micelles in many of their properties. Information on the aggregation of these molecules in aqueous systems and their interaction with other molecules in solution, can be obtained conveniently by laser Raman Spectroscopy. In the present paper Raman spectral studies on the hydrotrope sodium butyl monoglycol sulphate (NaBMGS), is presented and discussed with reference to spectral changes in solution and interaction with probe molecules.
\end{abstract}

Keywords. Hydrotropes; solubilising agents; Laser Raman spectroscopy; butyl monoglycol sulphate.

\section{Introduction}

It is well-known that surfactants, micelles, and hydrotropes belong to a class of compounds which are important in many chemical and physical processes such as catalysis, direct energy conversion, micro emulsions, solubilisation etc. A clear understanding of the structure-property relations in these systems is very useful in many applications, so that tailor-made systems can be prepared to suit specific applications. Since these are all used in aqueous systems, a convenient method for understanding their properties and interactions at a molecular level is the technique of Raman spectroscopy. Laser Raman spectroscopy can be used not only for direct structure-property correlations, but also to identify subtle interactions, through frequency and intensity changes of vibrational bands. Techniques like depolarised Raman scattering, difference Raman spectroscopy and time-resolved Raman studies can be employed to study vibrational dephasing and reorientational motions, intermolecular interactions, and transport properties. In the present paper the results of Raman and infrared spectroscopy studies on typical hydrotropes are presented and discussed with reference to some of these applications.

\section{Materials and methods}

Pure sodium butyl monoglycol sulphate (NaBMGS) was obtained from Huls (Germany) and used as such. All other reagents were GR grade. Double-distilled water was used

\footnotetext{
* For correspondence
} 
for preparation of solutions. All solutions were prepared and filtered immediately before running the spectrum.

Raman spectra were recorded with a Spex Ramalog Raman spectrometer interfaced by us to a Wipro p. c. The $514.5 \mathrm{~nm} \mathrm{Ar}^{+}$laser line was used for excitation. Laser power at the sample was kept about $100-200 \mathrm{mw}$. A spectral slit width of $2 \mathrm{~cm}^{-1}$ was employed for all scans. To record a spectrum, the starting frequency, step size, time of signal collection at each step, and spectral range are fed to the computer. The computer then positions the spectrometer to the starting frequency, collects the counts for the given time, increments frequency by one step and repeats data collection, until end of scan is reached. Several scans were averaged when required, and all data processing like peak finding, generation of difference spectra etc. were done on the same computer.

The spectrometer was calibrated using lines from a uranium hollow cathode.

\section{Results and discussion}

The aim of the present studies is three-fold. First, to see whether sufficient spectral changes are observable under different conditions of concentration; second, to infer the reasons for such changes; and third, to correlate the spectral changes to interactions with other molecules. Our preliminary results indicate that important information can be obtained in all these areas from the Raman spectral studies.

In figure 1, we show the Raman spectra in the $\mathrm{CH}$ stretching region of NaBMGS, for the solid, the saturated solution and a $0.5 \mathrm{M}$ solution. It can be seen that while the spectrum of the saturated solution resembles that of the solid very closely, the $0.5 \mathrm{M}$ solution differs from the other two both in frequency and relative intensity. The $\mathrm{CH}$ stretch region of long-chain molecules is known to be highly sensitive to packing (Snyder et al 1978; Kartha et al 1984), and the results thus show that at higher concentrations, $\mathrm{NaBMGS}$ tends to aggregate in solution with a packing similar to that of the solid. Earlier results (D Balasubramanian, V Srinivas, V G Gaikar and M M Sharma, unpublished) by some of the authors of the present work have shown that NaBMGS starts to show aggregational behaviour above $0.8 \mathrm{M}$ concentration.

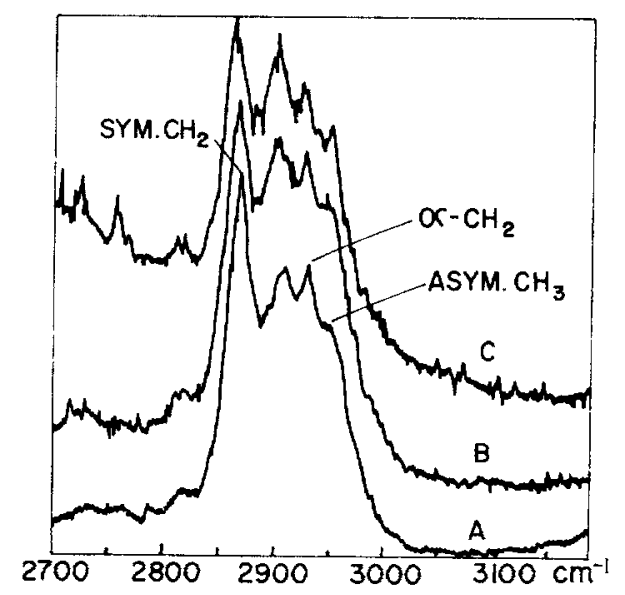

Figure 1. $\mathrm{CH}$-stretch-sodium butyl monoglycol sulphate. A-0.5 M solution; B-saturated solution; C-solid. 
The present studies also support this conclusion but the precise nature of the aggregation can be understood only after detailed studies of the other spectral regions as well and of the crystal structure of the solid.

Interaction of the hydrotrope with other systems can be studied using probe molecules and techniques like fluorescence, EPR etc. We have used $\mathrm{CH}_{3} \mathrm{CN}$ as a probe molecule and studied the interaction between the probe and the hydrotrope. In the Raman spectrum of pure acetonitrile, the $\mathrm{C}-\mathrm{N}$ stretching mode appears at $2251 \cdot 5 \mathrm{~cm}^{-1}$ and is highly polarised. Our spectra show that the parallel polarised component has an FWHM (full width at half maximum) of less than $4 \mathrm{~cm}^{-1}$, while the perpendicular component has a width of more than $12 \mathrm{~cm}^{-1}$. To a first approximation, these widths give the vibrational dephasing and reorientational relaxation times (Griffiths 1977), given by $1 /\left(2 \pi C \Delta v_{\frac{1}{2}}\right)$, where $\Delta v_{\frac{1}{2}}$ is the HWHM (half width at maximum). In pure acetonitrile the above widths indicate these times to be about 3 and 1 ps respectively.

When acetonitrile is mixed with water, in addition to a shift of the peak frequency to $2254.5 \mathrm{~cm}^{-1}$, there is a considerable change in the FWHM of the parallel polarised component of the Raman band, its value increasing to about $11 \mathrm{~cm}^{-1}$. This shows the much larger interaction between water and $\mathrm{CH}_{3} \mathrm{CN}$, and the vibrational dephasing times have been reduced by a factor of at least 3 due to this interaction. The perpendicular component in this system has an FWHM of $13 \mathrm{~cm}^{-1}$, but no information can be obtained unless a detailed time-correlation function calculation is made, correcting for the isotropic scattering (Kartha and Kartha 1980). If now, instead of water we use a solution of NaBMGS, keeping the acetonitrile to water ratio the same as before, we see that the peak frequency shifts back to almost the original value $\left(2251.9 \mathrm{~cm}^{-1}\right)$ and the width of the parallel component is also reduced considerably $\left(<8 \mathrm{~cm}^{-1}\right)$. This is shown in figure 2 . A more clear picture of the change in the acetonitrile spectrum is shown by the difference spcectra in figure 3 . In this figure the shift in frequency from pure acetonitrile to the acetonitrile-water mixture



Figure 2. $\mathrm{CN}$-stretch $\left(\mathrm{CH}_{3} \mathrm{CN}\right)$. A-pure liquid; B-water solution $(1: 1 \mathrm{v} / \mathrm{v}) ; \mathrm{C}-\mathrm{CH}_{3} \mathrm{CN}+$ NaBMGS solution $(1: 1 \mathrm{v} / \mathrm{v})$. 


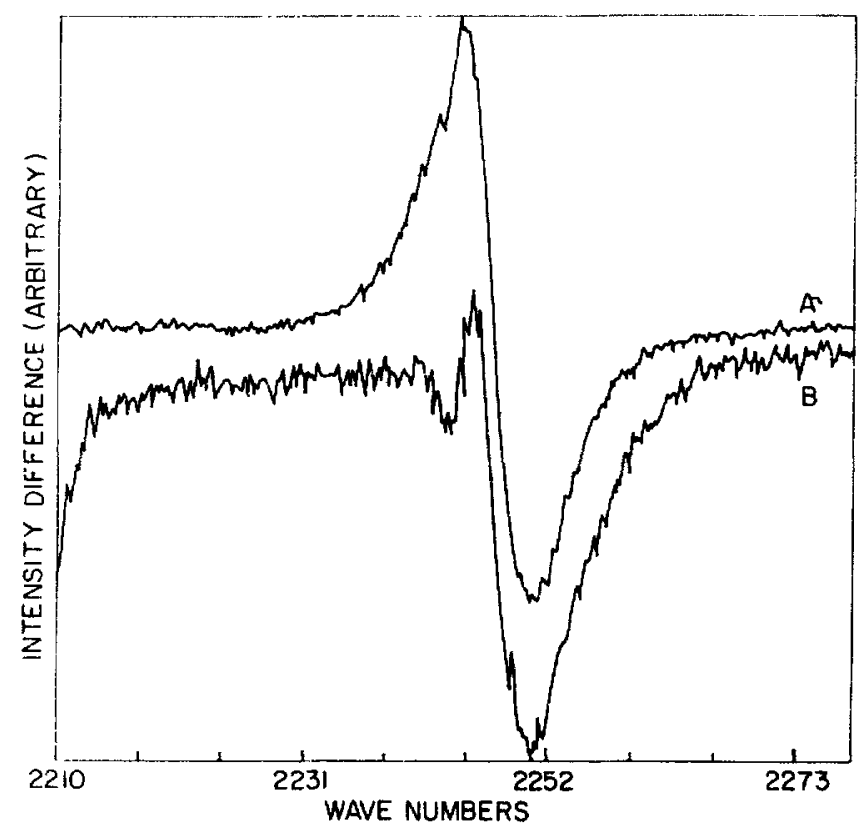

Figure 3. $\mathrm{CN}$-stretch $\left(\mathrm{CH}_{3} \mathrm{CN}\right)$. A-difference spectrum of pure $\mathrm{CH}_{3} \mathrm{CN}$ and $\mathrm{CH}_{3} \mathrm{CN}$ in water; $\mathrm{B}$-difference spectrum of pure $\mathrm{CH}_{3} \mathrm{CN}$ and $\mathrm{CH}_{3} \mathrm{CN}$ mixed with aqueous solution of NaBMGS.

Table 1. Raman data on the $v_{2}$ band of $\mathrm{CH}_{3} \mathrm{CN}^{2}$.

\begin{tabular}{|c|c|c|c|c|}
\hline \multirow{2}{*}{ System $^{b}$} & \multirow{2}{*}{$\begin{array}{c}v_{2} \begin{array}{c}\text { Frequency } \\
\left(\mathrm{cm}^{-1}\right)\end{array} \\
\end{array}$} & \multicolumn{3}{|c|}{ Half width $\left(\mathrm{cm}^{-1}\right)$ FWHM } \\
\hline & & $\Delta v_{\frac{1}{2}} \mathrm{~N}$ & $\Delta v_{\frac{1}{2}} \mathrm{PL}$ & $\Delta v_{\frac{1}{2}} \mathrm{PR}$ \\
\hline Pure liquid & $2251 \cdot 5$ & 4 & $3 \cdot 5$ & $12 \cdot 3$ \\
\hline $\mathrm{CH}_{3} \mathrm{CN}+\mathrm{H}_{2} \mathrm{O}$ & $2254 \cdot 5$ & 11 & 11 & $13 \cdot 3$ \\
\hline $\begin{array}{l}\mathrm{CH}_{3} \mathrm{CN}+\mathrm{Nabutyl} \\
\text { monoglycol sulphate } \\
50 \mathrm{~mol} \% \text { solution }\end{array}$ & 2251.9 & 8 & $8 \cdot 7$ & $14 \cdot 7$ \\
\hline $\begin{array}{l}\mathrm{CH}_{3} \mathrm{CN}+\mathrm{SDS} \\
\quad 60 \mathrm{~mol} \% \text { solution }\end{array}$ & $2254 \cdot 2$ & 10 & $10 \cdot 8$ & $13 \cdot 3$ \\
\hline
\end{tabular}

\footnotetext{
${ }^{a}$ All solutions are made up of equal volumes of $\mathrm{CH}_{3} \mathrm{CN}$ and surfactant solutions or water.

${ }^{\mathrm{b}}$ For pure acetonitrile and acetonitrile $+\mathrm{Na}$ butyl monoglycol sulphate the band shows considerable asymmetry. The half widths are taken to be twice the value of the HWHM of the side without asymmetry.

'The $\Delta v_{1}$ values are $\mathrm{N}$ - normal, PL - parallel polarisation, PR perpendicular polarisation.
}

can be clearly seen by the differential curve produced by subtracting the intensity of acetonitrile-water spectrum from that of pure acetonitrile. On the low frequency side of this curve we have the strong positive lobe, corresponding to the peak position of pure acetonitrile, while the high frequency side gives the strong negative lobe, showing the intense band in water solution. When NaBMGS is added to this system, the positive lobe almost completely disappears, while the negative lobe remains, showing 
that the band has now two components, one corresponding very closely to that of pure acetonitrile, while the other corresponds to the acetonitrile-water system. This clearly shows that in the hydrotrope solution, the acetonitrile is trapped in an environment similar to that in the pure liquid. This is possible only when there is aggregation of the hydrotrope molecules, and the $\mathrm{CH}_{3} \mathrm{CN}$ is held in a cavity formed by the hydrocarbon end of the hydrotrope so that $\mathrm{CH}_{3} \mathrm{CN}$ 'sees' only this hydrocarbon environment. Whether the aggregation is of micellar form or not cannot be decided without further studies using several probe molecules. Further work on these aspects, as well as the reorientational motion and relaxation of probe molecules is being carried out at present.

For comparison with hydrotropes, the $\mathrm{CN}$ band of acetonitrile was also studied in two other systems, namely the micellar system sodium dodecyl sulphate (SDS) and the reverse micellar system $\mathrm{AOT}-\mathrm{CCl}_{4}$-water. In very strong and very dilute solutions of SDS, the $\mathrm{CH}_{3} \mathrm{CN}$ band was almost identical to that in water, while intermediate solutions showed a splitting; in AOT, the $\mathrm{CH}_{3} \mathrm{CN}$ band was identical to that in $\mathrm{CCl}_{4}$, showing that the $\mathrm{CH}_{3} \mathrm{CN}$ is not attached to the water present. These results indicate that the environment of $\mathrm{CH}_{3} \mathrm{CN}$ in NaBMGS is different from that of both SDS and AOT. The results for the various systems are summarised in table 1.

In conclusion, it can be seen that Raman spectral studies show that in concentrated solution NaBMGS shows aggregation similar to that of the solid, and these aggregates can enclose additives in a hydrocarbon environment. Also, the aggregation appears to be different from that in micellar or reverse micellar systems.

\section{References}

Griffiths J E 1977 Vibrational spectra and structure (ed.) Durig J R (New York: Elsevier) vol. 6, chap. 5 Kartha V B and Kartha S B 1980 Proc. VII Int. Conf. Raman Spectroscopy (ed.) W F Murphy (Ottawa: National Research Council of Canada) pp. 500-501

Kartha V B, Leitch L C and Mantsch H H 1984 Can. J. Chem. 62128

Snyder R G, Hsu S L and Krimm S 1978 Spectrochim. Acta A34 395 